\title{
Spatial Subchondral Bone Density Reflecting Joint Loading of the Talus in Different Canidae
}

\author{
Walter B. Dingemanse ${ }^{1, \#}$ Ingrid M. L. V. Gielen ${ }^{2,3, \#}$ Henri J. J. van Bree ${ }^{4}$ Magdalena Müller-Gerbl ${ }^{5}$ \\ Nikola E. Krstić ${ }^{2}$ Marko B. Mitrović ${ }^{2}$ Duško S. Ćirović6 Mirjana V. Lazarević Macanović ${ }^{2}$
}

\footnotetext{
${ }^{1}$ Kennel \& Paddock Veterinary Rehabilitation and Hydrotherapy, James Lane, Grazeley Green, Berkshire, United Kingdom

2 Department of Radiology and Radiation Hygiene, Faculty of Veterinary Medicine, University of Belgrade, Belgrade, Serbia

${ }^{3}$ Department of Medical Imaging of Domestic Animals and Orthopedics of Small Animals, Faculty of Veterinary Medicine, Ghent University, Merelbeke, Belgium

${ }^{4}$ VetMedlmage, Erondegem, Belgium

${ }^{5}$ Department of Biomedicine, Institute of Anatomy, Basel University, Basel, Switzerland

6 Department of Animal Ecology and Zoogeography, Faculty of Biology, University of Belgrade, Serbia
}

Vet Comp Orthop Traumatol 2019;32:207-214.

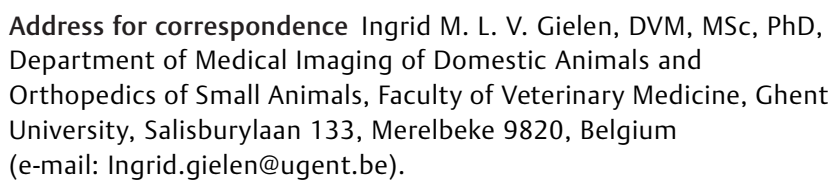

Address for correspondence Ingrid M. L. V. Gielen, DVM, MSc, PhD, Department of Medical Imaging of Domestic Animals and Orthopedics of Small Animals, Faculty of Veterinary Medicine, Ghent University, Salisburylaan 133, Merelbeke 9820, Belgium (e-mail: Ingrid.gielen@ugent.be).

\author{
Abstract \\ Keywords \\ - computer \\ tomographic \\ osteoabsorptiometry \\ - subchondral bone \\ density \\ - Canidae \\ - jackal \\ - wolf \\ - computed \\ tomography \\ - dogs \\ - joint biomechanics
}

Objective Subchondral bone density distribution can be used to study joint biomechanics non-invasively. Differences in joint loading between related species can aid in the understanding of joint loading and the development of certain types of orthopaedic pathology. This study was conducted to evaluate density distribution in the subchondral bone of the talus of different Canidae species, as a parameter reflecting the long-term joint loading in the tarsocrural joint.

Materials and Methods The tarsal joints of cadaveric dogs of different breeds were included, that is, German Shepherd $(n=5)$, Bouvier des Flandres $(n=3)$ and Labrador Retriever $(n=6)$.

Additionally, golden jackals $(n=5)$ (Canis aureus) and wolves $(n=5)$ (Canis lupus) were included. Consecutive computed tomography slices were made and the subchondral bone density distribution was evaluated using computer tomographic osteoabsorptiometry. Different breeds and species were visually compared.

Results Differences were found in the subchondral bone density distribution of the talus between breeds and between species (Canis familiaris, Canis lupus and Canis aureus).

Discussion and Conclusion Based on the density distribution, there are differences in loading conditions of the tarsocrural joint in different species of Canidae. The joint loading distribution is very similar between dogs of the same breed and within the same species. Although between-breed differences can be explained by conformational differences, the between-species differences remain subject to further research.

\footnotetext{
\# Contributed equally to the study.
}

received

May 23, 2018

accepted after revision

December 27, 2018
(C) 2019 Georg Thieme Verlag KG Stuttgart · New York
DOI https://doi.org/ 10.1055/s-0039-1685511. ISSN 0932-0814. 


\section{Introduction}

The integrity of the skeletal system is maintained by a continuous remodelling process that responds to mechanical forces and that results in the coordinated resorption and formation of skeletal tissue. This process occurs on a microscopical scale within bone tissue by basic multicellular units in which the cellular components are osteoclasts and osteoblasts. ${ }^{1}$ This process has been studied extensively and is known as Wolff's law. An important step leading to adaptation of bone to mechanical loading is the transduction of physical stimuli into biochemical factors that can alter the activity of the osteoblasts and osteoclasts. ${ }^{2}$ A key regulator of osteoblast and osteoclast activity is mechanical strain. The physical stimulus causing mechanical strain at the level of the subchondral bone is mainly joint loading, which includes two different aspects, that is, body weight and the forces exerted by musculotendon complexes. ${ }^{3}$ It is characterized by tensile and compressive stresses and plays an important role in cartilage and subchondral bone physiology. ${ }^{3,4}$ Since the evaluation of actual joint loading is very difficult in vivo, most studies focus on the morphological properties of bone, as they reflect the loading history. ${ }^{5-8}$ Using intra-articular pressure films ${ }^{9}$ or other in vitro methods, joint loading can be studied. ${ }^{10,11}$ However, these techniques require a certain degree of dissection, which will ultimately alter the joint kinematics, and by definition cannot be applied to living animals.

The density distribution of the subchondral bone is the result of functional adaptation and provides an opportunity for non-invasive research of joint loading. ${ }^{12,13}$ Bones continually adapt their mechanical properties as a response to changes in environment. ${ }^{3,14}$

As demonstrated in different species, the subchondral bone density distribution is highly correlated with joint loading and reflects the loading history of the joint. $8,12,15$ The main morphological properties determining the density of bones are bone porosity and the degree of mineralization ${ }^{13,16}$ and in radiographical techniques such as computed tomography (CT), these properties determine the output in Hounsfield units (HU). Therefore, the subchondral bone density distribution can be visualized and quantified using computer tomographic osteoabsorptiometry (CTOAM). ${ }^{8,15,17}$

Joint loading has been linked to the development of different types of orthopaedic conditions in dogs, such as osteochondritis, cranial cruciate ligament rupture and elbow dysplasia. ${ }^{17-21}$ In many orthopaedic conditions, a breed predisposition is seen, ${ }^{22,23}$ either due to genetic factors within the breed, ${ }^{24}$ or due to conformational traits such as joint angulations (although these are ultimately also influenced by genetics) influencing joint kinematics and joint loading. For instance, femoral angles have been described in different breeds and show significant differences. ${ }^{25}$ All over the world, dogs (Canis familiaris) are represented in numerous different breeds and have the greatest intra-species variation (or phenotypic disparity) of all mammals. ${ }^{26}$ This phenotypic disparity is also reflected in their locomotion characteristics such as joint kinematics and dynamics. ${ }^{27,28}$ This phenotypic disparity is likely to influence joint loading distribution, and thus subchondral bone density distribution.

In primates, different types of locomotor behaviours such as bipedalism, quadrupedalism and suspensory locomotion have been studied to evaluate limb-loading regimes. ${ }^{5,29}$ The diversity of limb posture can be related to different primate species and has affected the species-specific subchondral bone density distributions. ${ }^{29}$ Joint anatomy and joint biomechanics are the main contributors to joint loading distribution which are reflected in the subchondral bone density distribution.

Although the differences in joint loading distribution between dogs of different breeds will not be as big as that between bipedal and quadrupedal primate species, or even between knuckle-walk and palmigrade primates, ${ }^{5,13}$ the aim of this study was to compare the subchondral bone density distribution of the talus between different dog breeds as a reflection of their phenotypic disparity. Morphometric studies in the elbow joint of carnivores have shown differences in joint anatomy linked to hunting methods. ${ }^{30,31}$

Additionally, the grey wolf and the golden jackal were evaluated. The latter was included as the species that is a close relative to the domesticated dog, despite marked size differences. The morphological diversity of different dog breeds has a genetic base, linking different dog breeds and their common ancestors. ${ }^{32}$ The closest relative of the dog is the grey wolf (Canis lupus), followed by the coyote (Canis latrans), golden jackal (Canis aureus) and Ethiopian wolf (Canis simensis). ${ }^{32}$ The comparison of joint loading distribution between dogs and their close relatives can aid in the understanding of joint loading and joint pathology in dogs.

More specifically, differences in overall mean and maximum density were evaluated (null hypothesis-no difference in overall mean and maximum density), in addition to the location of the density maximum within the subchondral bone density distribution (null hypothesis-no difference in the location of the density maximum). These comparisons were made intra-breed, inter-breed and inter-species.

\section{Materials and Methods}

\section{Study Population}

In this study, cadaveric dogs of different breeds were included, that is, German Shepherd $(n=5 ; 3$ males, 2 females; 34.1 [9.7] kg), Bouvier des Flandres ( $n=3 ; 3$ males, weight 43.7 [8.7] $\mathrm{kg})$ and Labrador Retriever $(n=6 ; 4$ males, 2 females; 29.7 [5.2] kg), euthanatized for reasons unrelated to this study. The tarsal joints of golden jackals (Canis aureus) ( $n=5$, sex and weight unknown, on average 6-14 kg) and wolves (Canis lupus) (Eastern Europe, $n=5$, sex and weight unknown on average $35 \mathrm{~kg}$; cadaveric specimens) were included for comparison with the different dog breeds.

All specimens were deemed radiographically free of orthopaedic disease affecting the thoracic and pelvic limbs and lumbosacral joint and all joints were inspected and visually deemed clear of any gross orthopaedic pathology and cartilage lesions. 
All dogs were fresh cadavers that were scanned within 24 hours of euthanasia for reasons unrelated to this study. The wolf and jackal specimens had all tissues removed and were stored frozen until time of scanning.

\section{Image Acquisition}

The CT images were acquired from the tarsal joints using a four-slice helical CT scanner (Lightspeed Qx/i; General Electric Medical Systems, Milwaukee, Wisconsin, United States). The CT parameters were $120 \mathrm{kVp}$ and $300 \mathrm{mAs}$. Contiguous, $1.25-\mathrm{mm}$ collimated, transverse images were obtained in both a bone and a soft tissue reconstruction algorithm.

Left and right tarsal joints were scanned simultaneously, with the tarsal joints in extension, according to patient protocol and previous publications. ${ }^{17,33}$ Correct positioning was confirmed on the laterolateral and dorsoplantar scout view.

\section{Image Analysis}

The CT images were exported in DICOM format to commercially available software (Analyze 11.0, Biomedical Imaging Resource, Mayo Foundation, Rochester, Minnesota, United States), used to complete the CTOAM workflow. The workflow results in an articular surface representation of the underlying subchondral bone density and has been described in detail recently. ${ }^{17}$

Two visual representations (proximal and dorsal view, - Fig. 1) of the density distribution (densitogram) of each joint were created, which were further evaluated. The original volume can be rotated in all directions and the proximal view was reconstructed first to visually include the most proximal, medial and lateral border of the joint surface. The dorsal view was obtained by tilting the proximal view caudally by $90^{\circ}$.

For quantification purposes, the density values (in $\mathrm{HU}$ ) were converted to 8-bit values, that is, 256 density values, which were split equally over 8 bins, according to literature. ${ }^{13}$ Thus, each bin contains a range of 32 density values. A density maximum was defined as an area with density values in the two highest density bins of the densitogram. To quantify the density maxima, a $30 \times 30$ unit grid was projected onto the densitogram of the proximal and dorsal view. The grid edges were positioned to ensure that the entire joint surface could fit within. The number of units in



Fig. 2 Positioning of the grid on a proximal view of the talus and description of the subchondral bone density maximum by $x$ - and $y$ coordinates. Medial (M) and lateral (L) aspect are indicated.

each grid was kept the same, to standardize the coordinates of the density maxima. The density maxima were characterized by their $x$ - and $y$-coordinates (-Fig. 2).

\section{Statistics}

Using commercially available software (SPSS Statistics 22; IBM Inc., Somers, NY, United States), the location of the density maxima ( $x$ - and $y$-coordinates), the mean density and the maximum density was compared intra-breed, interbreed and inter-species. Data were evaluated using the analysis of variance and significance was set at $p<0.05$.

\section{Results}

\section{Subchondral Bone Density Values and Distribution of the Different Dog Breeds}

In mean and maximum density, no significant differences were seen between the Labrador Retriever and the German Shepherd on the proximal and dorsal view, but the Bouvier showed a significantly higher mean and maximum density (-Table 1).
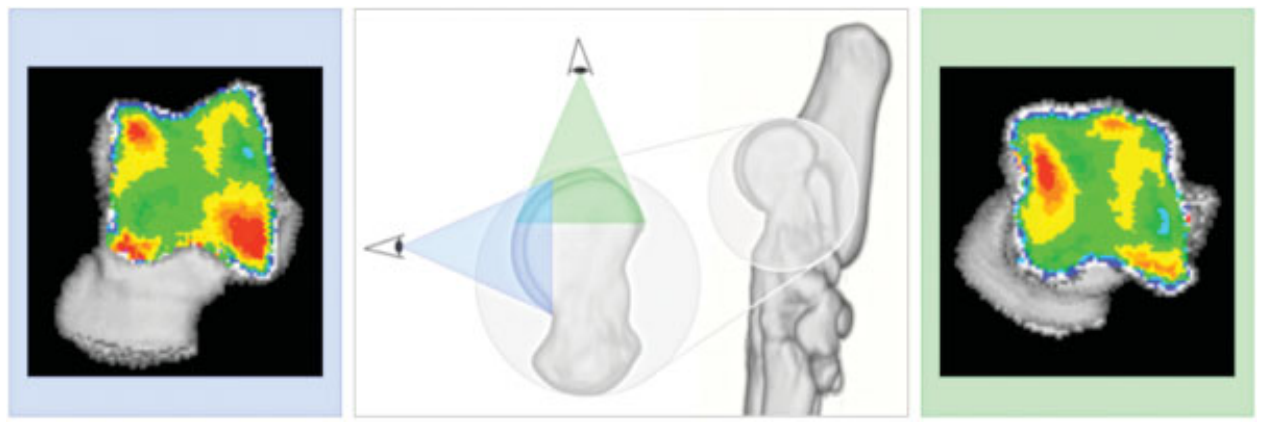

Fig. 1 Three-dimensional reconstruction of the tarsal and metatarsal bones of the right hindlimb, medial view. Line of sight for the two threedimensional reconstructions that are reconstructed from the segmented images, proximal view (green) and dorsal view (blue). The use of these two views provides full visualization of the trochlear ridges with the typical distribution shown for the proximal (right) and dorsal (left) view. 
Table 1 Summary of overall density of different dog breeds

\begin{tabular}{|l|l|l|l|l|}
\hline & & Labrador Retriever & German Shepherd & Bouvier des Flandres \\
\hline $\begin{array}{l}\text { Mean density } \\
\left(\mathrm{mg} \mathrm{HA} / \mathrm{cm}^{3}\right)\end{array}$ & Proximal view & $736.0(46.3)$ & $705.4(52.7)$ & $884.2(48.2)$ \\
\cline { 2 - 5 } & Dorsal view & $761.5(36.9)$ & $781.4(49.1)$ & $911.3(62.8)$ \\
\hline $\begin{array}{l}\text { Maximum density } \\
\left(\mathrm{mg} \mathrm{HA} / \mathrm{cm}^{3}\right)\end{array}$ & Proximal view & $1122.2(77.9)$ & $1084.4(61.5)$ & $1291.5(77.3)$ \\
\cline { 2 - 5 } & Dorsal view & $1186.6(78.0)$ & $1161.7(47.9)$ & $1351.7(81.4)$ \\
\hline
\end{tabular}

Abbreviation: SD, standard deviation.

Note: Mean density and maximum density (in $\mathrm{mg}$ hydroxyapatite $/ \mathrm{cm}^{3}$ ). Values displayed as mean (SD).

In the different breeds, different patterns of subchondral bone density were seen in the talus. In - Fig. 3, representative densitograms of the different breeds are shown. No significant differences in the location of subchondral bone density maxima were seen intra-breed on the proximal and dorsal view, indicating patterns were very similar between dogs of the same breed.

In the Labrador Retriever, two density maxima were seen, one proximal on the medial trochlear ridge and one distal on the lateral trochlear ridge. Even in the German Shepherds, two density maxima were present, but they were located at the distal aspect of the lateral and medial trochlear ridge. The Bouviers showed a large density maximum covering almost the entire lateral trochlear ridge.

There was a significant difference in the location ( $x$ - and $y$ coordinates) of the density maximum on the proximal view between all breeds ( $p$-Values $=0.029$ and 0.012 ). On the dorsal view, there was no significant difference in the location of the density maximum on the lateral trochlear ridge (0.498 and 0.655$)$. The location of the density maximum on the medial trochlear ridge differed significantly between all breeds ( $p$-Value $=0.871$, absent in the Bouviers). Differences in density maximum coordinates are illustrated in -Fig. 4.

\section{Subchondral Bone Density Distribution of the Grey Wolf and Golden Jackal}

In mean and maximum density, no significant differences were seen between the Labrador Retriever, grey wolf and golden jackal on the proximal and dorsal view. Representative densitograms of the talus of the grey wolf, golden jackal and Labrador Retriever (for comparison) are displayed in - Fig. 5.

At the proximal aspect of the wolf talus, two density maxima were seen, one on the medial trochlear ridge and other on the lateral trochlear ridge. An area with increased density was also present at the distal aspect of the lateral trochlear ridge. In the golden jackals, an overall high density was seen at the proximal aspect of both trochlear ridges, with a large density maximum located on the medial trochlear ridge. The distal aspect of the lateral trochlear ridge showed an increased density, whereas the medial trochlear ridge showed an area of very low density at the same level (-Table 2).

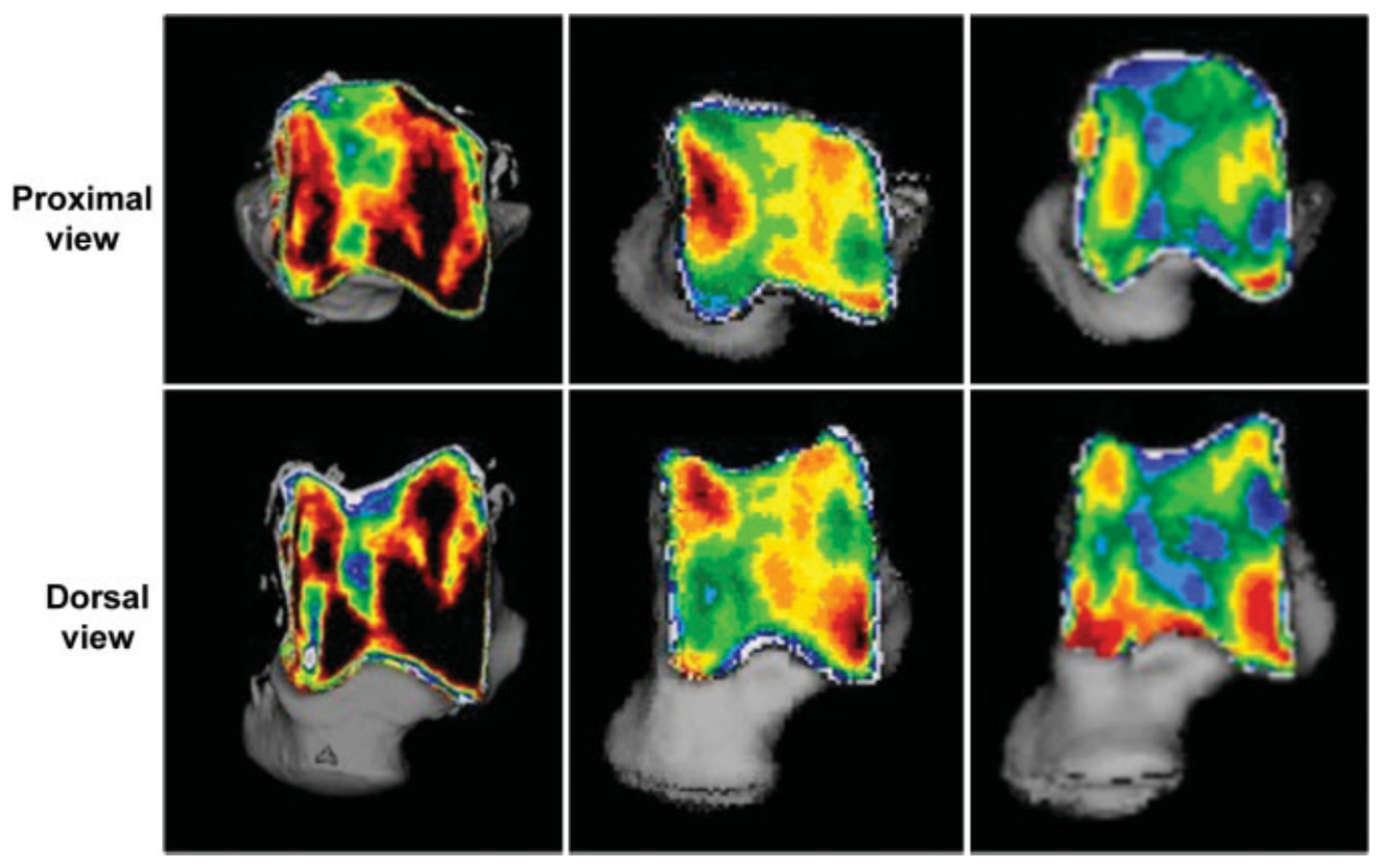

Fig. 3 Representative densitograms of different dog breeds: Bouvier des Flandres (left), Labrador Retriever (middle) and German Shepherd (right). 


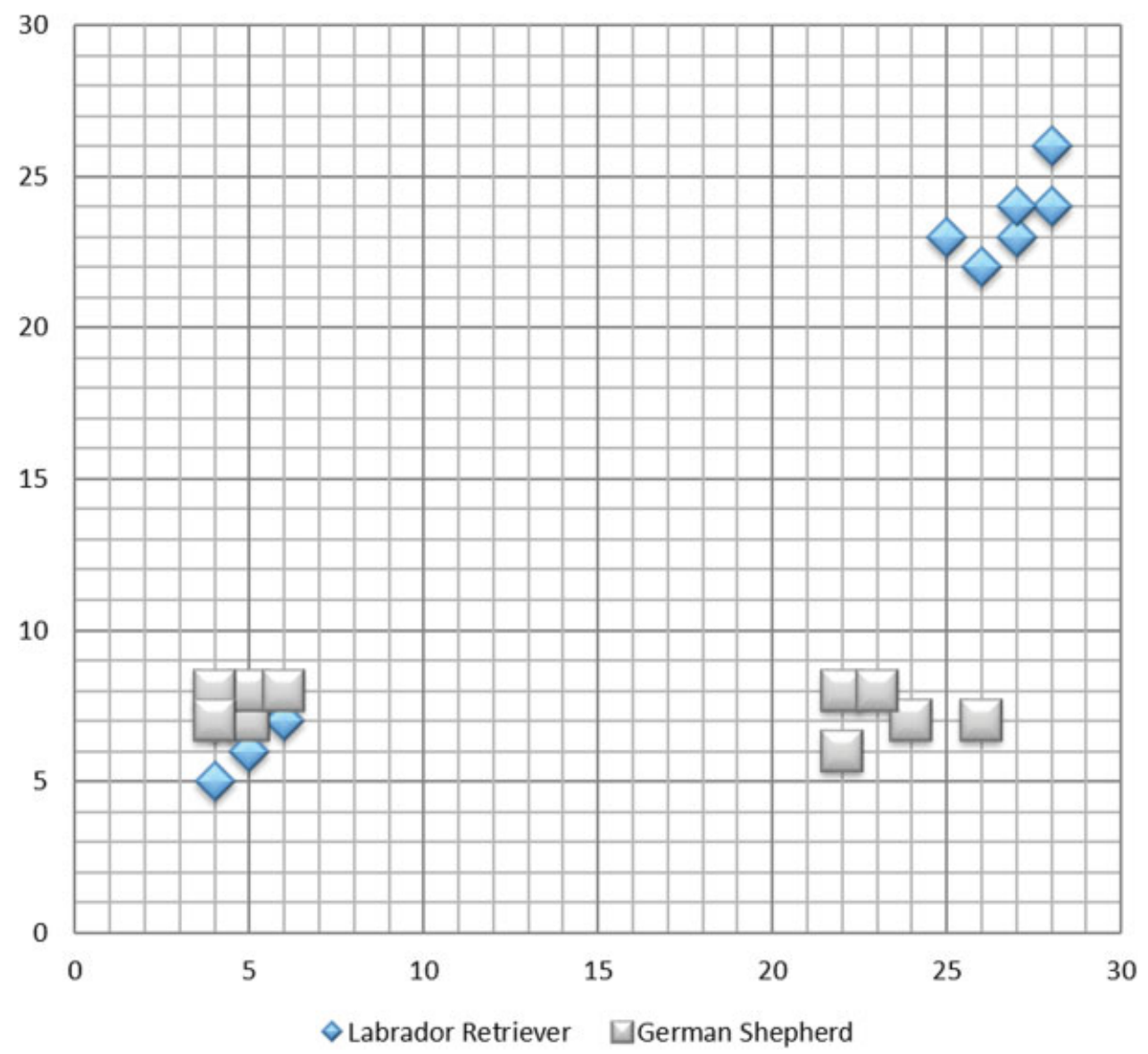

Fig. 4 Overview of the coordinates of the density maxima for the Labrador Retrievers and German Shepherds on the dorsal view.

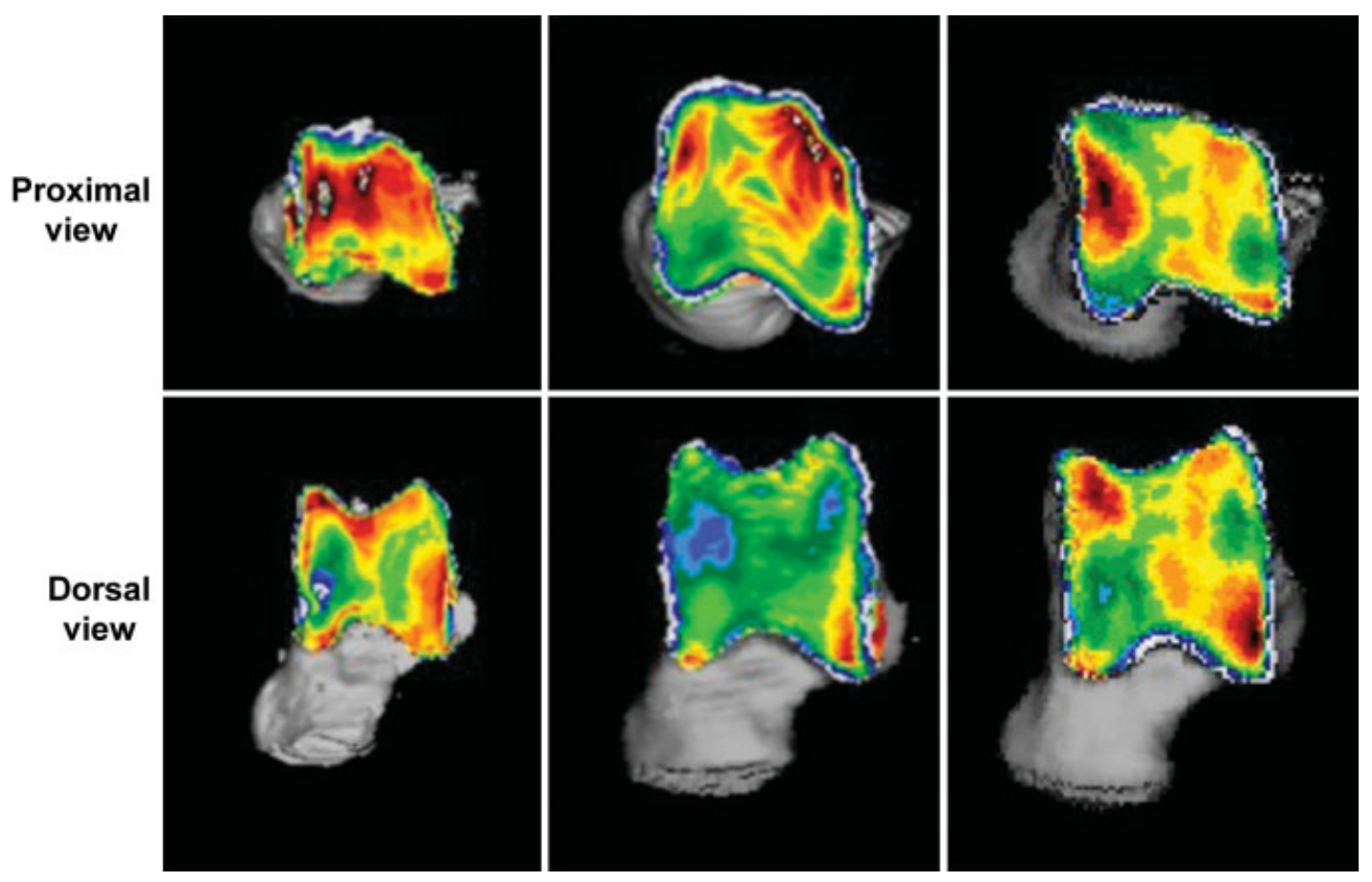

Fig. 5 Representative densitograms of the golden jackal (left), grey wolf (middle) and Labrador Retriever (right). 
Table 2 Summary of overall density of different Canidae

\begin{tabular}{|l|l|l|l|l|}
\hline & & Labrador Retriever & Grey wolf & Golden jackal \\
\hline \multirow{2}{*}{ Mean density $\left(\mathrm{mg} \mathrm{HA} / \mathrm{cm}^{3}\right)$} & Proximal view & $736.0(46.3)$ & $757.6(46.1)$ & $784.9(42.1)$ \\
\cline { 2 - 5 } & Dorsal view & $761.5(36.9)$ & $719.4(48.8)$ & $745.4(57.3)$ \\
\hline \multirow{2}{*}{ Maximum density $\left(\mathrm{mg} \mathrm{HA} / \mathrm{cm}^{3}\right)$} & Proximal view & $1122.2(77.9)$ & $1131.9(71.4)$ & $1189.6(82.5)$ \\
\cline { 2 - 5 } & Dorsal view & $1186.6(78.0)$ & $1088.4(83.4)$ & $1091.4(79.7)$ \\
\hline
\end{tabular}

Abbreviation: SD, standard deviation.

Note: Mean density and maximum density (in $\mathrm{mg}$ hydroxyapatite $/ \mathrm{cm}^{3}$ ). Values displayed as mean (SD).

There was a significant difference in the location ( $x$ - and $y$ coordinates) of the density maximum on the proximal view between the Labrador Retriever and the wolf $(p$-Value $=0.001)$. On the dorsal view, there was no significant difference in the location of the density maximum on the lateral trochlear ridge (0.319 and 0.878$)$. The location of the density maximum on the medial trochlear ridge differed significantly between Labrador Retriever and the wolf as it was absent in the wolf. There was no significant difference between the Labrador Retriever and the golden jackal ( $p$-Value $=0.609$ ).

\section{Discussion}

In this study, the spatial characteristics of the subchondral bone density of the talus were compared between different dog breeds, wolves and golden jackals. In case of the intra-breed, both null hypotheses could not be rejected, whereas in the interbreed and inter-species the null hypotheses were rejected. For the domestic dog (Canis familiaris), the density distribution differs inter-breed and is very similar intra-breed. This is consistent with previous reports on the subchondral bone density distribution in the talus of healthy Labrador Retrievers. ${ }^{17}$

The most notable difference in density distribution was seen between the Labrador Retrievers and German Shepherds. In the latter, two density maxima were located at the distal aspect of the medial and lateral trochlear ridge. The pelvic limb angulation, commonly seen in this breed, can potentially explain the subchondral bone density distribution described in this study (-Fig. 6), although more research is needed to establish the relationship between joint angulation and articular loading

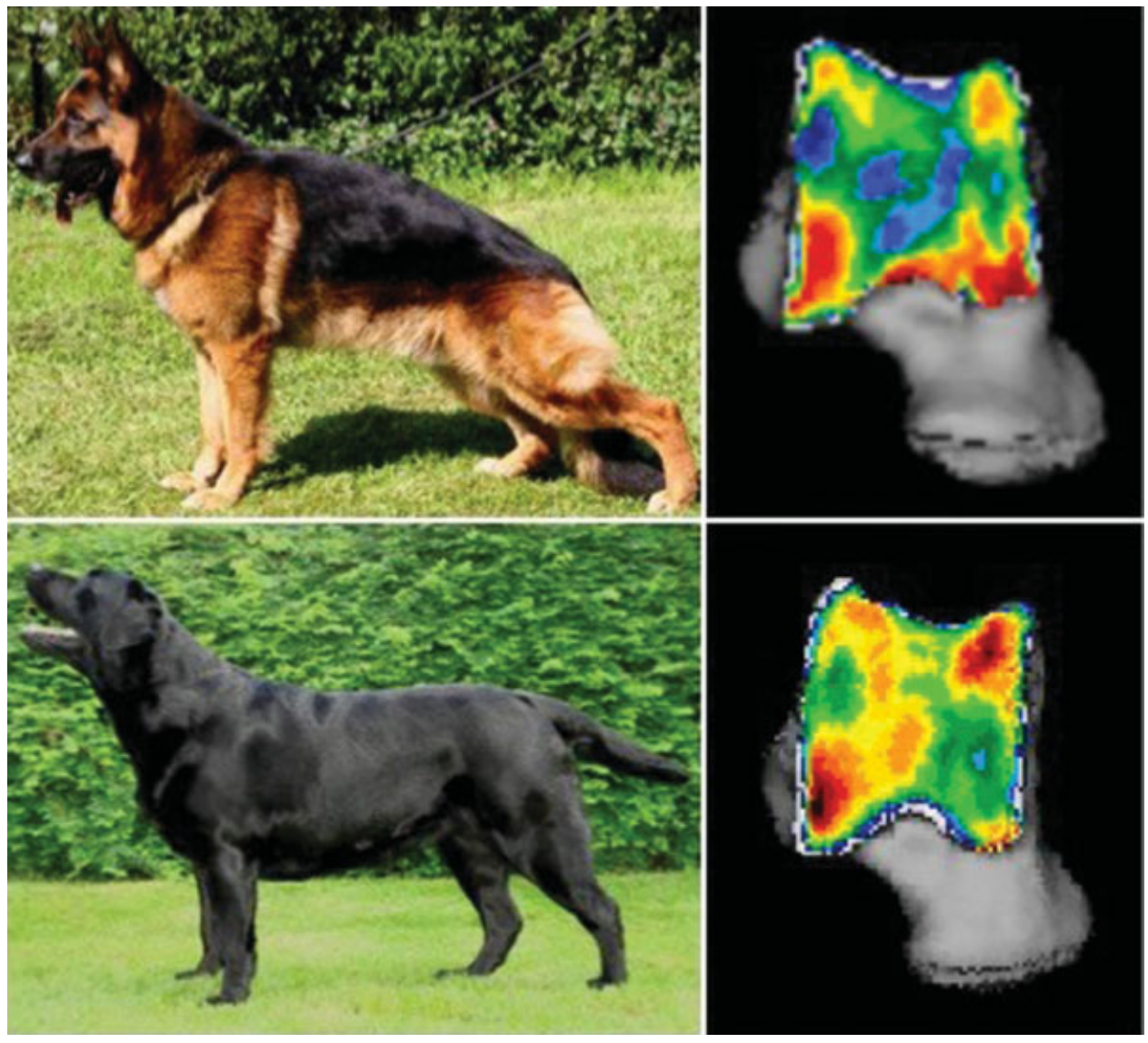

Fig. 6 Comparison of the body conformation and subchondral bone density distribution of the talus between the German Shepherd (top) and Labrador Retriever (bottom). Note the extreme angulation of the tarsal joint in the German Shepherd, typical for dogs of this breed. 
patterns. Additionally, the density maximum in the Bouvier was located on the lateral trochlear ridge, and a maximum on the medial trochlear ridge, as seen in the Labrador Retriever is absent. The authors speculate that this could potentially be linked with trochlear ridge morphology as the lateral trochlear ridge was visually more pronounced in the Bouvier, but a morphometric analysis was not attempted in this study and remains subject to further research.

Breed differences have also been reported for joint kinematics and kinetics. ${ }^{27,28}$ Gross differences exist in the joint moments and power in the hip, stifle and hock joint between Labrador Retrievers and Greyhounds. ${ }^{16}$ This will reflect on the muscle work done by the pelvic limb musculature, which is an important component of joint loading, in addition to the effect of bodyweight and velocity. The absence of significant differences in mean and maximum density highlights that interbreed differences in subchondral bone density are to be found in the density distribution, not the absolute density values, and are therefore most likely related to joint loading distribution.

The subchondral bone density distribution of two of the closest relatives of the domestic dog, that is, the wolf and the golden jackal, also showed regional differences between the different Canidae. More interestingly, there were no significant differences in the location of the density maxima between the Labrador Retriever and golden jackal, indicating that the loading distribution pattern is likely to be similar despite marked size differences. How these differences in subchondral bone density distribution correlate to hindlimb angulation, and locomotion behaviour remains subject to further research.

The functional anatomy of joints can be used to study joint biomechanics and to reveal differences in gait and limb use in specific species. ${ }^{5,29,34}$ Studies on the evolutionary aspect of locomotion have used the general form-function relationship as the central mechanism in bone biology. ${ }^{34}$ Gross differences have been described between bipedal and quadrupedal primates ${ }^{29}$ and between arboreal and terrestrial marsupials. ${ }^{34}$ Conformational adaptations related to specific breeds will alter joint loading patterns and may in turn have an effect on the incidence of orthopaedic pathology. In the elbow joint, morphometric differences exist between breeds, and between different Canidae species based on their way of sourcing food. ${ }^{30}$ Although there are currently no data available on differences in joint kinematics and kinetics in the specific breeds used in this study, the differences in subchondral bone density distribution indicate potential differences in joint loading patterns. The relationship between differences in loading pattern, locomotion and orthopaedic pathology remains subject to further research.

\section{Limitations}

The exact age of the jackals and wolves was unknown and may significantly alter subchondral bone density. However, in previous study focusing on age-related changes, there were only changes in mean and maximal density values and no significant differences in subchondral bone density distribution. The density distribution pattern remains the same, indicating that joint loading conditions are likely to remain similar in healthy individuals. ${ }^{35}$

\section{Conclusion and Future Research}

Significant differences in subchondral bone density distribution pattern exist between different breeds included in this study, although mean and maximum density values are similar. This study focused on only three breeds and in future work more breeds are needed to be included to fully understand the effect of joint morphology on subchondral bone density distribution and the development of orthopaedic pathology.

The increase in studies investigating joint biomechanics and breed-specific parameters highlights the need for breed-specific biomechanical data. ${ }^{22}$ The use of CTOAM in the evaluation of subchondral bone density distribution can play a central role in canine orthopaedic research, as CT data are more readily available than specific kinematic or kinetic datasets. By studying and comparing different breeds, joint pathology can be evaluated in the light of joint loading and the adaptations within different breeds. This will aid in our understanding of joint loading and may help to explain the breed predisposition seen in many orthopaedic conditions in domestic dogs.

\section{Author Contribution}

W. Dingemanse and I. Gielen contributed to conception of study, study design, acquisition of data and data analysis and interpretation. $\mathrm{H}$. van Bree contributed to conception of study and study design. M. Muller-Gerbl contributed to study design and acquisition of data. N. Krstić, M. Mitrović and D. Ćirović contributed to acquisition of data. M. Lazarević Macanović contributed to study design and data analysis and interpretation. All authors drafted, revised and approved the accepted manuscript.

\section{Conflict of Interest}

None of the authors of this article has a financial or personal relationship with other people or organizations that could inappropriately influence or bias the content of the article.

\section{Acknowledgments}

The authors like to thank our Serbian colleagues Dr. Ivan Rakić, Dr. Ljubomir Ćurčin and Dr. Jòzsef Özvegy for their help in obtaining the specimen.

\section{References}

1 Parfitt AM. The bone remodeling compartment: a circulatory function for bone lining cells. J Bone Miner Res 2001;16(09): 1583-1585

2 Vatsa A, Breuls RG, Semeins CM, Salmon PL, Smit TH, KleinNulend J. Osteocyte morphology in fibula and calvaria-is there a role for mechanosensing? Bone 2008;43(03):452-458

3 Frost HM. The Utah paradigm of skeletal physiology: an overview of its insights for bone, cartilage and collagenous tissue organs. J Bone Miner Metab 2000;18(06):305-316

4 Turner $\mathrm{CH}$. Three rules for bone adaptation to mechanical stimuli. Bone 1998;23(05):399-407

5 Ebel CM, Prodinger PM, Mühlhofer H, Müller-Gerbl M, Linsenmaier U, Putz R. Morphological adaptation of the tarso-metatarsal joints onto load transmission in the foot. Surg Radiol Anat 2010; 32(02):107-113 
6 Eckstein F, Jacobs CR, Merz BR. Mechanobiological adaptation of subchondral bone as a function of joint incongruity and loading. Med Eng Phys 1997;19(08):720-728

7 Frost HM. A 2003 update of bone physiology and Wolff's Law for clinicians. Angle Orthod 2004;74(01):3-15

8 Müller-Gerbl M, Putz R, Kenn R. Demonstration of subchondral bone density patterns by three-dimensional CT osteoabsorptiometry as a noninvasive method for in vivo assessment of individual long-term stresses in joints. J Bone Miner Res 1992;7(Suppl 2):S411-S418

9 Coughlin KM, Peura GD, Fleming BC, Hallock S, Beynnon BD. In vivo loads in the medial compartment of the rabbit knee. Clin Biomech (Bristol, Avon) 2005;20(09):1007-1009

10 Mason DR, Schulz KS, Fujita Y, Kass PH, Stover SM. Measurement of humeroradial and humeroulnar transarticular joint forces in the canine elbow joint after humeral wedge and humeral slide osteotomies. Vet Surg 2008;37(01):63-70

11 Preston CA, Schulz KS, Kass PH. In vitro determination of contact areas in the normal elbow joint of dogs. Am J Vet Res 2000;61(10): $1315-1321$

12 Madry H, van Dijk CN, Mueller-Gerbl M. The basic science of the subchondral bone. Knee Surg Sports Traumatol Arthrosc 2010;18 (04):419-433

13 Carlson KJ, Patel BA. Habitual use of the primate forelimb is reflected in the material properties of subchondral bone in the distal radius. J Anat 2006;208(06):659-670

14 Currey JD. The design of mineralised hard tissues for their mechanical functions. J Exp Biol 1999;202(Pt 23):3285-3294

15 Müller-Gerbl M, Putz R, Hodapp NH, Schulta E, Wimmer B. Computed tomography-osteoaboorptiometry: a method of assessing the mechanical condition of the major joints in a living subject. Clin Biomech (Bristol, Avon) 1990;5(04):193-198

16 van der Meulen MC, Jepsen KJ, Mikić B. Understanding bone strength: size isn't everything. Bone 2001;29(02):101-104

17 Dingemanse W, Müller-Gerbl M, Jonkers I, Vander Sloten J, van Bree $\mathrm{H}$, Gielen I. Subchondral bone density distribution of the talus in clinically normal Labrador Retrievers. BMC Vet Res 2016;12:56

18 Cook JL. Cranial cruciate ligament disease in dogs: biology versus biomechanics. Vet Surg 2010;39(03):270-277

19 Dickomeit MJ, Böttcher P, Hecht S, Liebich HG, Maierl J. Topographic and age-dependent distribution of subchondral bone density in the elbow joints of clinically normal dogs. Am J Vet Res 2011;72(04):491-499

20 Dingemanse WB, Van Bree HJ, Duchateau L, Gielen IM. Comparison of morphological and clinical features between medial and lateral trochlear ridge talar osteochondrosis dissecans in dogs. Vet Surg 2013; 42(03):340-345
21 Alves-Pimenta S, Ginja MM, Fernandes AM, Ferreira AJ, Melo-Pinto $\mathrm{P}$, Colaço B. Computed tomography and radiographic assessment of congruity between the ulnar trochlear notch and humeral trochlea in large breed dogs. Vet Comp Orthop Traumatol 2017;30(01):8-14

22 Colborne GR. Bringing canine biomechanics research out of the dark ages. Vet J 2007;173(03):469-470

23 Griffon DJ. A review of the pathogenesis of canine cranial cruciate ligament disease as a basis for future preventive strategies. Vet Surg 2010;39(04):399-409

24 Coopman F, Broeckx B, Verelst E, et al. Combined prevalence of inherited skeletal disorders in dog breeds in Belgium. Vet Comp Orthop Traumatol 2014;27(05):395-397

25 Tomlinson J, Fox D, Cook JL, Keller GG. Measurement of femoral angles in four dog breeds. Vet Surg 2007;36(06):593-598

26 Moody JA, Clark LA, Murphy KE. 2006. Canine History and Breed Clubs. Cold Spring Harbor Monograph. Series 44:1;2006

27 Agostinho FS, Rahal SC, Miqueleto NS, Verdugo MR, Inamassu LR, El-Warrak AO. Kinematic analysis of Labrador Retrievers and Rottweilers trotting on a treadmill. Vet Comp Orthop Traumatol 2011;24(03):185-191

28 Colborne GR, Innes JF, Comerford EJ, Owen MR, Fuller CJ. Distribution of power across the hind limb joints in Labrador Retrievers and Greyhounds. Am J Vet Res 2005;66(09):1563-1571

29 Nowak MG, Carlson KJ, Patel BA. Apparent density of the primate calcaneo-cuboid joint and its association with locomotor mode, foot posture, and the "midtarsal break.". Am J Phys Anthropol 2010;142(02):180-193

30 Figueirido B. Phenotypic disparity of the elbow joint in domestic dogs and wild carnivores. Evolution 2018;72:1600-1613

31 Andersson KI. Elbow-joint morphology as a guide to forearm function and foraging behaviour in mammalian carnivores. Zool J Linn Soc 2004; 142:91-104

32 Lindblad-Toh K, Wade CM, Mikkelsen TS, et al. Genome sequence, comparative analysis and haplotype structure of the domestic dog. Nature 2005;438(7069):803-819

33 Gielen I, van Bree H, Van Ryssen B, De Clercq T, De Rooster H. Radiographic, computed tomographic and arthroscopic findings in 23 dogs with osteochondrosis of the tarsocrural joint. Vet Rec 2002;150(14):442-447

34 Carlson KJ, Jashashvili T, Houghton K, Westaway MC, Patel BA. Joint loads in marsupial ankles reflect habitual bipedalism versus quadrupedalism. PLoS One 2013;8(03):e58811

35 Dingemanse W, Müller-Gerbl M, Jonkers I, Sloten JV, van Bree H, Gielen I. A prospective follow up of age related changes in the subchondral bone density of the talus of healthy Labrador Retrievers. BMC Vet Res 2017;13(01):57 